\section{CANCER ANEUPLOIDY IS ASSOCIATED WITH A DISTINCT TUMOR IMMUNE MICROENVIRONMENT AND IMPACTS OUTCOMES TO IMMUNE CHECKPOINT INHIBITION IN NONSQUAMOUS NON-SMALL CELL LUNG CANCER}

${ }^{1}$ Joao Victor Alessi', ${ }^{1}$ Biagio Ricciuti, ${ }^{1} Y$ vonne Lin-Liu, ${ }^{1}$ Hersh Gupta, ${ }^{2}$ Xinan Wang, ${ }^{1}$ Giuseppe Lamberti, ${ }^{1}$ Gonzalo Recondo, 'Victor Vaz, ${ }^{1}$ Adriana Barrichello, 'Mizuki Nishino, ${ }^{1}$ Andrew Cherniack, ${ }^{1}$ James Lindsay, ${ }^{1}$ Bijaya Sharma, ${ }^{1}$ Kathleen Pfaff, ${ }^{1}$ Kristen Felt, ${ }^{3}$ Scott Rodig, ${ }^{1}$ Mark Awad. ${ }^{1} D F C I$, Boston, MA, USA; ${ }^{2}$ Harvard School of Public Health, Boston, MA, USA; ${ }^{3}$ Brigham and Women's Hospital, Boston, MA, USA

Background Cancer aneuploidy, an unbalanced number of chromosomes, is associated with somatic mutation rate, expression of proliferative genes, and altered immune signaling. Whether aneuploidy correlates with a distinct tumor immunophenotype or impacts clinical outcomes to immune checkpoint inhibitors (ICIs) in NSCLC is unclear.

Methods Among nonsquamous NSCLCs which underwent targeted next-generation sequencing, we retrospectively quantified aneuploidy using the fraction of chromosomal arm alterations (FAA), defined as the number of aneuploid chromosome arms divided by the number of chromosome arms assessed. An unbiased recursive partitioning algorithm was used to investigate an FAA level which best discriminated responders from non-responders to ICIs. Multiplexed immunofluorescence to quantify CD8, FOXP3, and PD-1-positive cell counts, as well as PD-L1 expression was performed on a separate cohort of nonsquamous NSCLCs to determine differences in tumor immune cells subsets according to FAA levels.

Results Among 1426 nonsquamous NSCLCs identified, FAA increased along with the increase of pathologic stage, and was highest among tumors harboring EGFR mutations and RET fusions, and lowest among those with KRAS, BRAF, and MET mutations. FAA inversely correlated with PD-L1 expression levels, and positively correlated with tumor mutational burden (TMB) (figure 1A-D). Among 281 NSCLCs treated with ICIs, the median FAA was significantly lower among patients with a partial response to ICI compared to those with stable or progressive disease $(0.11$ vs $0.21, \mathrm{P}=0.006)$. A fractional aneuploidy level of 0.06 (representing the lowest quartile of FAA) was identified as an optimal cutpoint to discriminate responders from non-responders to ICI. Compared to pts with an FAA $>0.06(\mathrm{~N}=212)$, pts with FAA $\leq 0.06(\mathrm{~N}=69)$ had a significantly higher ORR $(42.0 \%$ vs $19.3 \%, \mathrm{P}<0.001)$, longer median progression-free survival (mPFS 6.8 vs 3.2 months, HR: $0.64, \mathrm{P}=0.004$ ), and longer median overall survival (mOS 24.8 vs 13.8 months, HR: $0.65, \mathrm{P}=0.012$ ) with ICIs (figure 2). After adjusting for performance status, PD-L1 expression, TMB, and line of treatment, FAA retained a significant association with improved PFS (HR: $0.66, \mathrm{P}=0.018$ ) and OS (HR: $0.66, \mathrm{P}=0.041$ ) to immunotherapy. FAA had no impact on clinical outcomes among pts who received first-line platinum doublet chemotherapy without ICI (figure 3). Among 239 nonsquamous NSCLCs profiled by multiplex immunofluorescence, cancers with a low FAA ( $\leq 25$ th percentile) were significantly enriched in CD8 $+\mathrm{T}$ cells and had a higher CD8 + to FOXP3 + ratio compared to those with high FAA ( $>25$ th percentile) (figure 4$)$.
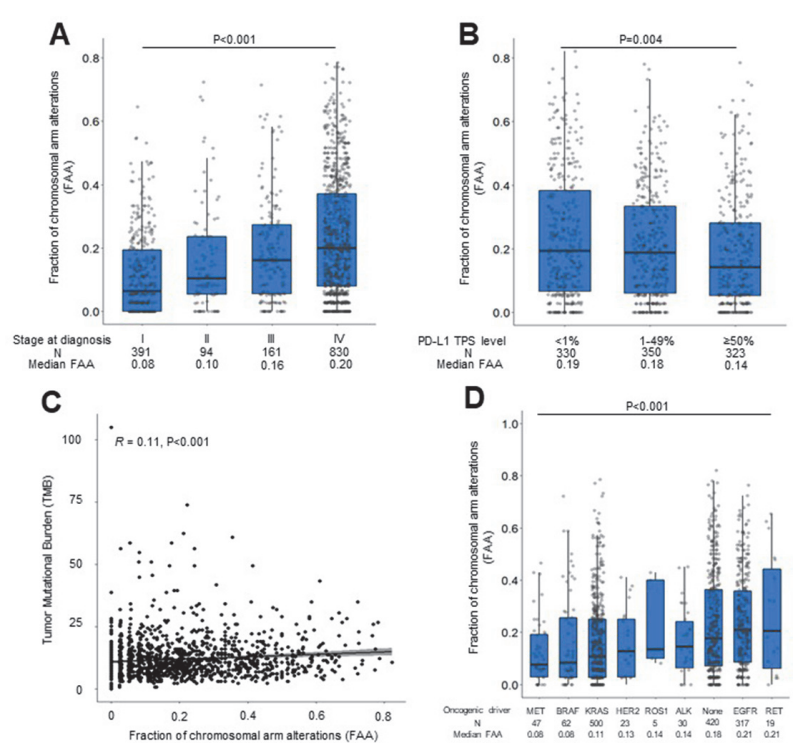

Abstract 67 Figure 1 (A) Median fraction of chromosomal alterations (FAA) are shown for stages I, II, III, and IV NSCLCs. (B) Tumors with negative $(<1 \%)$, low $(1-49 \%)$, and high PD-L1 tumor proportion score (TPS) $(\geq 50 \%)$ expression. (C) Pearson's correlation coefficient between FAA and tumor mutational burden (TMB). (D) Median FAA distribution across a set of 8 targetable driver mutations (ALK, BRAF, EGFR, HER2, KRAS, MET, RET and ROS1) and none identified alteration.
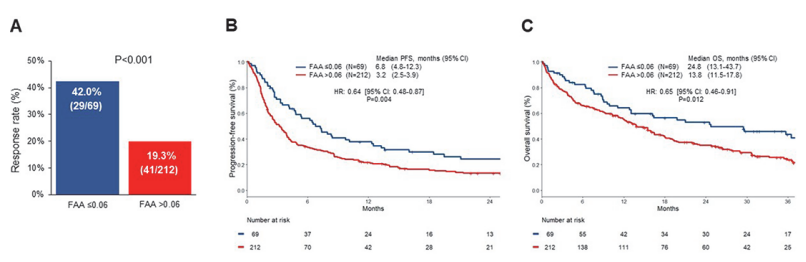

Abstract 67 Figure 2 (A) Objective response rate, (B) progressionfree survival, and $(C)$ overall survival, in patients with a fraction of chromosomal arm alterations (FAA) $\leq 0.06$ versus $>0.06$ in the immunotherapy-cohort.

Stage IV nonsquamous NSCLC First-line platinum doublet treated cohort
A

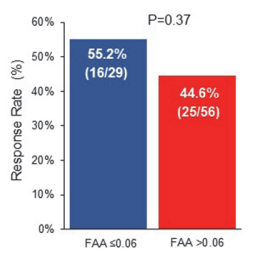

B

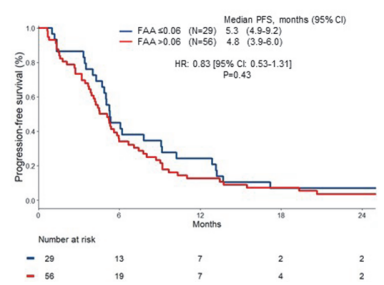

Abstract 67 Figure 3 (A) Objective response rate and (B) progression-free survival (PFS) in patients with a fraction of chromosomal arm alterations (FAA) $\leq 0.06$ versus $>0.06$ in the chemotherapy-cohort. 


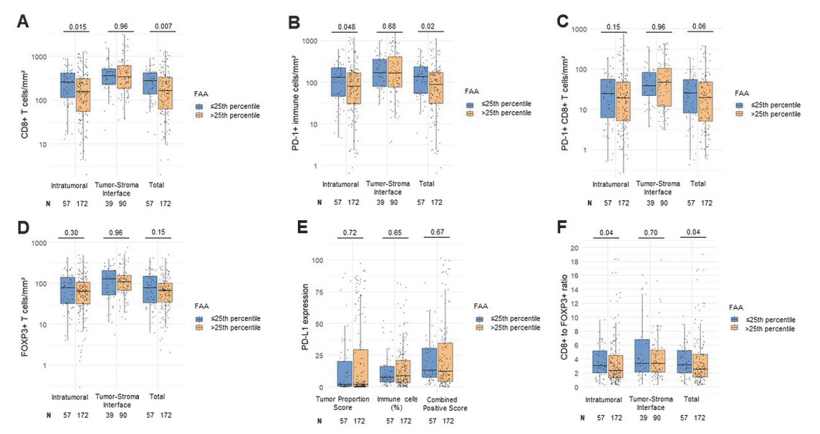

Abstract 67 Figure 4 (A) CD8+, (B) PD-1+, (C) PD-1+CD8+ (D), FOXP3+ cells/mm2, and (E) PD-L1 distribution in nonsquamous NSCLCs with an fraction of arm-level altered (FAA) low ( $\leq 25$ th percentile) versus high (>25th percentile). (F) CD8+ to FOXP3+ ratio in tumors with FAA low versus FAA high.

Conclusions Nonsquamous NSCLCs with low aneuploidy have a distinct immune microenvironment and more favorable outcomes to ICIs.

http://dx.doi.org/10.1136/jitc-2021-SITC2021.067 